\title{
Evaluación no invasiva de frecuencia respiratoria con dispositivo "dalí"
}

\author{
María Carolina Cabrera Schulmeyer ${ }^{1,2}$, Rocio Arancibia ${ }^{2}$
}

Introducción: La monitorización respiratoria del paciente sometido a un procedimiento quirúrgico e interpretación son la base del que hacer del anestesiólogo, permite diagnosticar, planificar y tratar las consecuencias fisiológicas en el paciente. La depresión respiratoria es una complicación del perioperatorio que se define como una frecuencia respiratoria menor a 8 respiraciones por minuto, cuando no es posible de despertar y/o saturación de $\mathrm{O}_{2}<88 \%$. Episodios críticos de depresión respiratoria se han reportado hasta en un 0,8 - 6,9\% de los pacientes en unidades de recuperación anestésica y otros estudios estiman que depresión respiratoria entendida como frecuencia respiratoria menor a 10 respiraciones por minuto por más de 2 minutos podría ocurrir en más del 50\% de los casos. Por lo tanto, se trata de un problema frecuente, con complicaciones asociadas y potencialmente prevenibles. El objetivo de este trabajo es validar el dispositivo Dalí como monitor de frecuencia respiratoria no invasivo, comparándolo con el conteo visual.

Material y Métodos: Se estudiaron a pacientes mayores de 18 años en la unidad de Recuperación anestésica que fueron sometidos a anestesia general o sedación, se les instaló dispositivo Dalí en región infranasal y se comparó con el análisis visual de la frecuencia respiratoria. Los resultados fueron analizados con software Python y se calcularon medidas de desempeño (sesgo, precisión y exactitud, además del gráfico de concordancia Bland-Altman), teniendo como referencia el conteo visual.

Resultados: Se estudiaron a 47 pacientes, 24 mujeres y 23 hombres con un promedio de edad de
$62,88 \pm 12,65$ y $49,91 \pm 15,48$ respectivamente, un peso promedio de $66,87 \pm 10,85 \mathrm{~kg}$ y $84,04 \pm 13,4$ $\mathrm{kg}$ y estatura promedio de 1,6 y 1,75 mts respectivamente, que fueron sometidos a procedimientos quirúrgicos, urológicos y traumatológicos principalmente, con anestesia general o sedación, en la unidad de recuperación. Se comparó la medición de frecuencia respiratoria mediante Dalí versus conteo visual, obteniéndose como resultado una precisión de 89,2\% con un intervalo de confianza del $95 \%$ en la predicción de la frecuencia respiratoria, con una diferencia de - 3,25 $y+2,19$ respiraciones por minuto.

Conclusiones: El dispositivo Dalí, demostró ser un monitor de frecuencia respiratoria no invasivo eficaz. Y preciso en la predicción de la frecuencia respiratoria de los pacientes sometidos a anestesia general o sedación en la unidad de recuperación anestésica, en comparación al conteo visual. Contar con un monitor de frecuencia respiratoria no invasivo, eficaz podría ser muy útil para prevenir episodios de depresión respiratoria.

\section{Referencias}

1. Overdyk FJ, Carter R, Maddox RR, Callura J, Herrin $A E$, Henríquez $C$. Continuous oximetry/capnometry monitoring reveals frequent desaturation and bradypnea during patient-controlled analgesia. Anesth Analg. 2007 Aug;105(2):412-8.

2. Dahan A, Aarts L, Smith TW. Incidence, reversal, and prevention of opioid-induced respiratory depression. Anesthesiology. 2010 Jan;112(1):226-38.

Hospital FACH, Chile.

Universidad de Valparaíso, Chile. 